\title{
Reconstruction of Branching Surface and Its Smoothness by Reversible Catmull-Clark Subdivision
}

\author{
Kailash Jha \\ Assistant Professor, Deptt. of Mechanical Engineering \\ \& Mining Machinery Engineering, \\ Indian School of Mines University, Dhanbad-826004, Jharkhand, India \\ kailash_jha@hotmail.com
}

\begin{abstract}
In the current research a new algorithm has been developed to get surface from the contours having branches and a final smooth surface is obtained by reversible Catmull-Clark Subdivision. In branching, a particular layer has more than one contour, corresponds with the contour at the adjacent layer. The layer having more than one contour is converted into a $3 \mathrm{D}$ composite curve by inserting points between the layers. The points are inserted in such a way that the center of contours should merged to the center of the contours at the adjacent layer. This process is repeated for all layers having branching problems. In the next step, 3D composite curves are converted into different polyhedrons by the help of the contours at adjacent layers. Number of control points at different layer for contours and $3 \mathrm{D}$ curves may not be the same, in this case a special polyhedron construction technique has been developed. The polyhedrons are subdivided using reversible Catmull-Clark subdivision to give a smooth surface.
\end{abstract}

Keywords: Catmull-Clark subdivision, branching surface, incompatible curves, reconstruction.

\section{Introduction}

In the present work, an algorithm has been developed to construct a three-dimensional surface from contours at different layers which may have branching problem and the required smooth surface is obtained with the help of the reversible Catmull-Clark subdivision. In the reversible Catmull-Clark subdivision level of smoothness is given by an integer value for required rendering. Construction of 3D surface from 2D contours is very important for CAD (Rapid prototyping, NC machining), Medical imaging and Geographical Information System. Technologies such as magnetic resonance imaging (MRI), computed topography (CT), and ultrasound imaging allow measurements of internal properties of objects to be obtained in a nondestructive fashion.

The points on the 2D contours are measured slice-by-slice. A slice may have more than one independent closed contour. These contours may correspond to one or more contours at adjacent slice. This situation is termed as branching, which is described in this work. The set of planes generating the slices are usually parallel to each other and may not be equi-spaced along any axis through the object. Once these slices have 
been obtained, the goal is to enable a human to easily visualize in 3D. Many algorithms have been developed for this purpose, but they can be classified into two categories, Volume Rendering Methods and Surface Reconstruction Methods. Volume rendering is used to show the characteristics of interior of the solid. In surface rendering, a geometrical representation is used to model the object or structure to be visualized based on original data such as edge, mesh, polygon, triangle or pixel. The present work focuses on surface reconstruction method. Generation of 3D surface from 2D contours has four basic steps: (a) Correspondence, (b) Tiling, (c) Branching and (d) Generation of surface.

(a) The correspondence problem involves finding the correct connections between the contours of adjacent slices. In the present formulation the correspondence problem is known which can be obtained from [1].

(b) Tiling means using slice chords to triangulate the strip lying between contours of two adjacent slices into tiling triangles. A slice chord connects a vertex of a given contour to a vertex of the contour in an adjacent slice. Each tiling triangle consists of exactly two slice chords and one contour segment. Details of tiling can be obtained from $[1,2]$. In the current work focus is given on branching problems. Our approach does not require tiling because it is based on construction of polyhedrons.

(c) A branching problem occurs when a contour in a layer corresponds to more than one contour in an adjacent layer.

(d) Solution of branching problem results polygonal surface which are smoothened by reversible Catmull-Clark subdivision.

There are three types of branching problems: (1) One-to-one, (2) One-to-many and (3) Many-to-many. One-to-one problem has been solved by several researchers based on minimizations of energy, twist and curvature and different tiling techniques which are shown in Fig 1a. One-to-many problem is the one in which at least one layer must have more than one contour and have correspondence with the contour at adjacent layers which is shown in Fig 1b. Many-to-many problem is stated as $m$ contours at $i$-th layer and $n$ contours at $(i+1)$ th layer and they are corresponding to each other. This problem can be solved by combining many one-to-many branching problems. Fig. 1c shows the Many-to-many branching problem. In the present technique, control points of curves at layer having branching problem are taken at a time and converted into 3D curve by linear merging of geometrical centers of the contours toward the center of contours at adjacent layer. For example, two contours $C_{1}$ and $C_{2}$ are shown

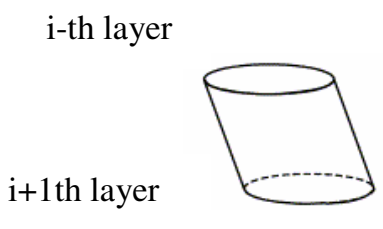

(a)

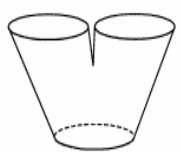

(b)

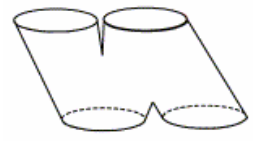

(c)

Fig. 1. Different type of branching: (a) One-to-one b; (b) Many-to-one; (c) Many-to-Many 
in Fig. 2 at layer $i$ having geometrical centers at $g_{1}$ and $g_{2}$ respectively, which are merged linearly to the geometrical center $\left(g_{3}\right)$ of adjacent contour $C_{3}$ at $(i+1)$ th layer. Lines parallel to $g_{1} g_{3}$ are drawn from all the control points of curves $C_{1}$ and similarly lines parallel to $\mathrm{g}_{2} \mathrm{~g}_{3}$ are drawn from control points of curve $\mathrm{C}_{2}$. The intersection point having highest value of $\mathrm{y}$-coordinate is considered for construction of 3D curves which is point $d$ in Fig 2. $\underline{\text { ad }}$ and $\underline{b d}$ are parallel to $g_{1} g_{3}$ and $g_{2} g_{3}$ respectively and intersect at highest value of $\mathrm{y}$-coordinate to give 3 -D curve, which is comprised of $\underline{\mathrm{db}}$, $C_{2}, \underline{\mathrm{bd}}, \underline{\mathrm{da}}, C_{1}$ and ad respectively. Once 3-D curve is obtained and the remaining contours at other layers do not have any branches, then the polyhedrons are constructed according to present formulation given in section 4 and the final 3-D surface is obtained by reversible Catmull-Clark subdivision, otherwise same process is repeated. Fig. 2 shows the contour points as well as other additional points. The current branching problem has been solved for the known correspondence and starting points for the contours at different layers. A complex 3D contour is generated for layer having branching problem, which converts many-to many branching problems into oneto-one branching problems.

Polyhedrons are constructed for all the pairs of adjacent layers once there is no branching problem. If contours are given as the points and constraints then the control polygons can be obtained by the references $[3,4]$ using energy based curve approximation.

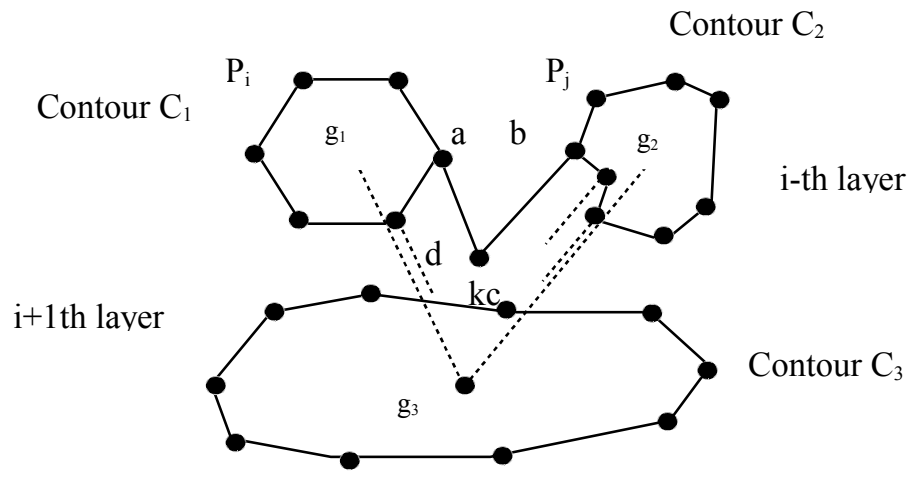

Fig. 2. Illustrated example to convert contours to a 3-D composite contour for a layer having two contours

A polygonal complex is a simple polygonal mesh whose structure depends on subdivision scheme used and whose limit of subdivision is a curve rather than a surface. The polygonal complexes are constructed for each of the polygons and polyhedron is constructed by connecting the polygon complexes side-by-side. Contours given are restricted to uniform cubic B-Spline curves [5,6] but it can be overcome by nonuniform technique given in [7]. The proposed technique is of order $\mathrm{O}(\mathrm{kn})$, where $\mathrm{n}$ is the number of cross section curves and $\mathrm{k}$ is the average number of control points per curve in the given sectional curves. 
The reversible Catmull-Clark subdivision has been developed in the present work to get smooth surface from a given polygons. It can be used for mesh generation. In graphics, shading and rendering improves the representation of surfaces and solids. The polygon construction technique in the current work is similar to $[5,6]$, which is not limited to the uniform B-Spline input curves. In the present work other type surface subdivision [8] can be incorporated. Reversible Catmull-Clark subdivision has been achieved in the present work, which is a unique feature of this work and is different from [6]. It also gives different stage of convergence of polygons toward the interpolating curves as well as smoothness of the surface. A boundary curve of a B-Spline patch is dependent only on the first three rows of the mesh defining the patch. It is same for the curve case where one end depends on the three control vertices. Present technique will be useful in medical imaging like modeling liver vessel tree, geological structure and stem of the tree.

Previous works are given in section 2. A brief overview of Catmull-Clark subdivision is given in section 3. Section 4 describes the current work. Results and discussions have been given in section 5. The research has been concluded in section 6 .

\section{Previous Works}

The literature that is devoted to many-to-one branching problem can be classified into four main families. The family of contour connection methods attempted to artificially render one-to-many problem into a one-to-one by connecting the disjoint contour with line [2] or triangulate facet bridge [9]. The first choice is applicable for simple cases, while the second constrains unnaturally the saddle points of the branching surface to lie on the plane containing the disjoint contours. The second family is based on introduction of intermediate contour, which splits the original problem into two problems one-to-one and a new one-to-many. The second problem is further simplified into $\mathrm{m}$ one-to-one problems. This idea has been proposed in $[10,11]$ and has been implemented in [12]. The family of partial contour connection and hole filling has been proposed in references $[1,13,14])$ is characterized by matching partially the disjoint contours with the single contours of the neighboring plane, thus leaving a number of holes which are finally filled in final step. Goodman et al., [15] has treated one-to-two case in which a single hole is filled by an approximated chosen hyperboloid.

Finally, the family of implicit schemes relies on the assumption that possesses implicit representation of contours composing the cross-sections. Then an implicit interpolate can be obtained by taking a convex combination of contour representation [16], or implying the distance function [12, 16]). Wang et al., [17] used Catmull-Clark subdivision for biorthogonal wavelet construction based on lifting scheme. Loop and Schafefer, [18] approximated Catmull-Clark subdivision surfaces by minimal set of bicubic patch. A brief overview of branching problems has been explained in reference [19], which involves skinning, trimming and hole filling. In reference [20] an algorithm has been developed to obtain branching surfaces by energy based skinning of compatible 2-D curves obtained by energy-based approximation. 


\section{Catmull-Clark Subdivision}

A polygonal mesh from which inner mesh can be obtained through the application of following rules basically defines a Catmull-Clark surface:

1. Each old face $f$ with $n$ vertices $\left(V_{i}\right)_{i<i<n}$, a new vertex $V_{f}$ can be generated at the centroid by:

$$
\mathrm{V}_{\mathrm{f}}=1 / \mathrm{n} \sum_{\mathrm{i}=1}^{\mathrm{n}} \mathrm{V}_{\mathrm{i}}
$$

2. For each of old edge e having two vertices $V_{1}$ and $V_{2}$ which is shared by two faces $\mathrm{f}$ and $\mathrm{g}$, a new vertex ve can be generated by:

$$
\mathrm{V}_{\mathrm{e}}=\left(\mathrm{V}_{1}+\mathrm{V}_{2}+\mathrm{V}_{\mathrm{f}}+\mathrm{V}_{\mathrm{g}}\right) / 4
$$

3. For each old vertex $V$ incident to $n$ edges $\left(e_{i}\right)$ and shared by $n$ faces $f_{i}$, a new vertex $\mathrm{V}_{\mathrm{v}}$ can be generated by:

$$
\mathrm{V}_{\mathrm{v}}=\alpha_{n} \sum_{i=1}^{n} V_{e i}+\beta_{n} \sum_{i=1}^{n} V_{f i}+\gamma_{n} V
$$

Where $V_{e i}$ (respectively $V_{f i}$ ) is the vertex generated from the edge $e_{i}$ (respectively the face fi), and the weight $\alpha_{n}, \beta_{n}$ and $\gamma_{n}$ are given by:

$$
\alpha_{n}=\beta_{n}=1 / \mathrm{n}^{2}, \quad \gamma_{n}=(\mathrm{n}-2) / \mathrm{n}
$$

The limiting curve of a Catmull-Clark polygonal complex can be determined in a piecewise manner. Basic formulation for Catmull-Clark subdivision has been given in [21]. Surface subdivision is based on rules and the given input polygons. Fig 3 shows the polygon and the subdivided polygons have been shown in Fig 4 along with the modified vertices, edge points and face point.

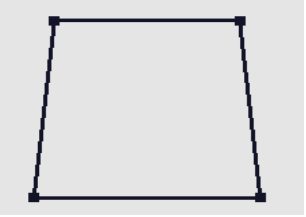

Fig. 3. Constructed polyhedron

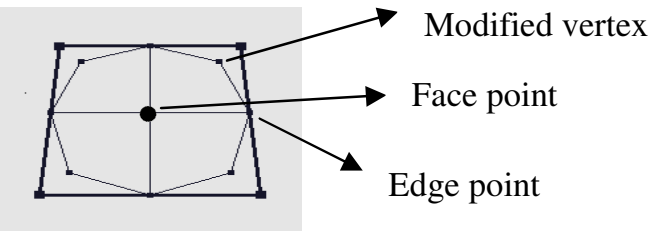

Fig. 4. Subdivided polyhedron with face points and vertices

\section{Current Work}

The present technique is based on construction of polyhedron from the generated 3D composite curves and polygons. Two or more set of control polygons are given at a layer which correspond to the control polygons of curves at adjacent layer. If the control points of the contours are not given, energy based approximation $[3,4]$ can be 
used to find their value. The first task is to calculate the center of each closed polygons and find slope of the line joining the centers of corresponding contours. In Fig 2, $\mathrm{g}_{1}, \mathrm{~g}_{2}$ and $\mathrm{g}_{3}$ are the CGs of the control polygons and $\mathrm{g}_{1} \mathrm{~g}_{3}$ and $\mathrm{g}_{2} \mathrm{~g}_{\underline{3}}$ are the line joining their centers. An algorithm has been developed to find out points $\left(\mathrm{P}_{\mathrm{i}}, \mathrm{P}_{\mathrm{j}}\right)$ in given curves for which the intersection of line parallel to $g_{1} g_{3}$ and passes through point $p_{i}$ and line parallel to $\mathrm{g}_{2} \mathrm{~g}_{3}$ and passes through $\mathrm{q}_{\mathrm{j}}$ having highest value of $\mathrm{y}$-coordinate intersection. Points $\mathrm{P}_{\mathrm{i}}, \mathrm{P}_{\mathrm{j}}$ and the intersection point $\mathrm{d}$ have been shown in Fig 2. Once the intersection point is obtained which is between the contours having correspondence, the closest point from this point at the adjacent layer is determined which, is the point $k c$ in Fig 2. With reference to these two points $(\mathrm{d}, \mathrm{kc})$, polyhedrons are constructed according to algorithm given in section 4.1. If the number of control points in the $3 \mathrm{D}$ composite curves is not the same as the number of control points at adjacent layer of contour then they are considered as the incompatible curves, which are solved by the technique given in ref $[5,6]$. After having the correct polyhedrons, the smooth surfaces are obtained by reversible subdivision of the polyhedrons (Catmull-Clark Subdivision), which is explained in Section 2. In the present work, reversible subdivision techniques have been adopted to go for the required smooth surface. Level of smoothness can be given by an integer value. Once the level of smoothness is given by the user, the corresponding surface will be displayed on the screen.

\subsection{Construction of Polyhedrons for Branching Contours}

The construction of surface polyhedron is done in such a way that a final control mesh will be obtained by connecting the resulting complexes side-by-side. This construction is divided into two phases:

(a) Reversible subdivision phase, (b) decomposition phase

These terminologies have been explained in references [5, 6]

\subsubsection{Reversible Subdivision Phase}

In this phase the given control polygons $\left(\mathrm{M}_{\mathrm{i}}\right.$ and $\mathrm{M}_{\mathrm{j}}$ ) (see Fig 5) of input curves are subdivided recursively until one of the subdivided control polygon does not have one edge. There are $n$ and $m$ vertices in control polygons $M_{i}$ and $M_{j}$ respectively. The ends of the polygons are joined together to make closed polygon. This closed polygon is again divided into two polygons by connecting at points where its Euclidian distance is minimum. Fig 5 shows the connection. This phase is also called virtual primitive phase. Fig 6 shows a primitive face, several such faces will be the output of the reversible subdivision phase.

Recursive subdivision can be illustrated by the following points:

1. $\mathrm{V}_{\mathrm{o}}, \mathrm{V}_{\mathrm{n}} \mathrm{V}_{\mathrm{m}}$ and $\mathrm{W}_{\mathrm{m}}$ are the boundary vertices

2. If $m$ and $n$ are one stop

3. Associated vertices $\mathrm{V}_{\mathrm{p}}$ and $\mathrm{W}_{\mathrm{q}}$ are calculated by shortest distance criterion

4. Subdivision is continued recursively for divided polygons

This process terminates with a set of virtual primitives faces, where each such face $f$ is represented by the pairs ( $\mathrm{vf}, \mathrm{wf})$ with $\mathrm{vf}$ and $\mathrm{wf}$ are the two set of vertices $\mathrm{V}_{\mathrm{f}}=$ $\left(\mathrm{V}_{\mathrm{i}}\right) \mathrm{a} \leq \mathrm{i} \leq \mathrm{a}+\mathrm{r}$ and $\mathrm{W}_{\mathrm{f}}=\left(\mathrm{W}_{\mathrm{i}}\right) \mathrm{a} \leq \mathrm{i} \leq \mathrm{a}+\mathrm{s}$ characterized by the following properties: 
1. Either $r$ and $s$ is equal to 1

2. The vertices $\mathrm{v}_{\mathrm{a}}$ and $\mathrm{w}_{\mathrm{b}}$ are the closest to $\mathrm{v}_{\mathrm{a}+\mathrm{r}}$ and $\mathrm{w}_{\mathrm{b}+\mathrm{s}}$ respectively among all possible pairs $\left(\mathrm{V}_{\mathrm{i}}, \mathrm{W}_{\mathrm{i}}\right)$ of the set $\left(\mathrm{V}_{\mathrm{f}} \mathrm{X} \mathrm{W}_{\mathrm{f}}\right)$

A primitive face $F$ can be defined by sequence $T$ from the vertices $M_{i}$ and another sequence $B$ from the vertices $\mathrm{M}_{\mathrm{j}}$. One of the sequences must have two vertices. Let $\mathrm{B}$ be the sequence having two vertices

$$
\begin{aligned}
& \mathrm{B}=\left\{\mathrm{W}_{1}, \mathrm{~W}_{2}\right\} \\
& \mathrm{T}=\left\{\mathrm{V}_{1}, \mathrm{~V}_{2} \quad \mathrm{~V}_{\mathrm{k}}\right\}
\end{aligned}
$$

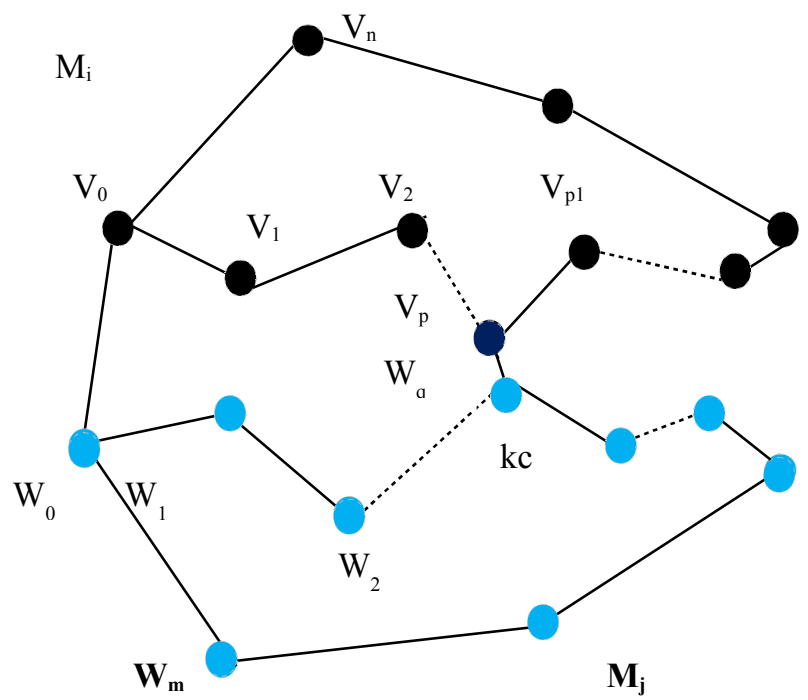

Fig. 5. Illustration for recursive subdivision

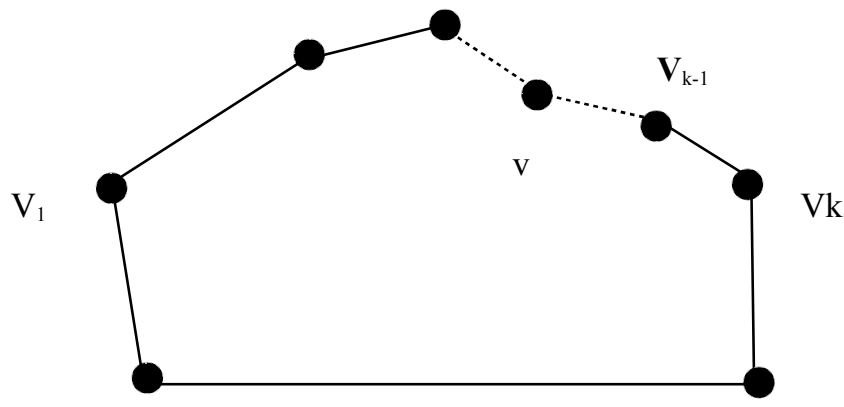

$\mathrm{W}_{1}$

$\mathrm{W}_{2}$

Fig. 6. Primitive face obtained by recursive subdivision for decomposition 


\subsubsection{Decomposition Phase}

In this phase the primitive faces are decomposed into several actual faces. Number of the faces is equal to number of vertices $(k)$ in T. First the k-2 vertices are created on B between $\mathrm{W}_{1}$ and $\mathrm{W}_{2}$ in same proportion distance as the vertices of $\mathrm{V}_{\mathrm{k}}$. $\mathrm{K}$ vertices $\left(\mathrm{V}_{\mathrm{k}}{ }^{\prime}\right)$ are created in between the two set of vertices $\mathrm{B}$ and $\mathrm{T}$ in 2: 1 ratio of distance and similar two vertices $\left(\mathrm{W}_{1}{ }^{\prime}\right.$ and $\left.\mathrm{W}_{2}{ }^{\prime}\right)$ are created on line $\underline{\mathrm{V}}_{1} \underline{\mathrm{W}}_{1} \underline{ }_{\text {and }} \underline{\mathrm{V}}_{\underline{n}} \underline{\mathrm{W}_{\mathrm{n}}}$ in $1: 2$ ratio of distance. Constructed polyhedrons and surfaces are shown in Fig 7. $\mathrm{V}_{1} \mathrm{~V}_{2} \mathrm{~V}_{2}{ }^{\prime} \mathrm{V}_{1}$ ' is a typical constructed polyhedron in Fig 7. Constructed polyhedrons for non-branching incompatible curves and has been shown in Fig 8.

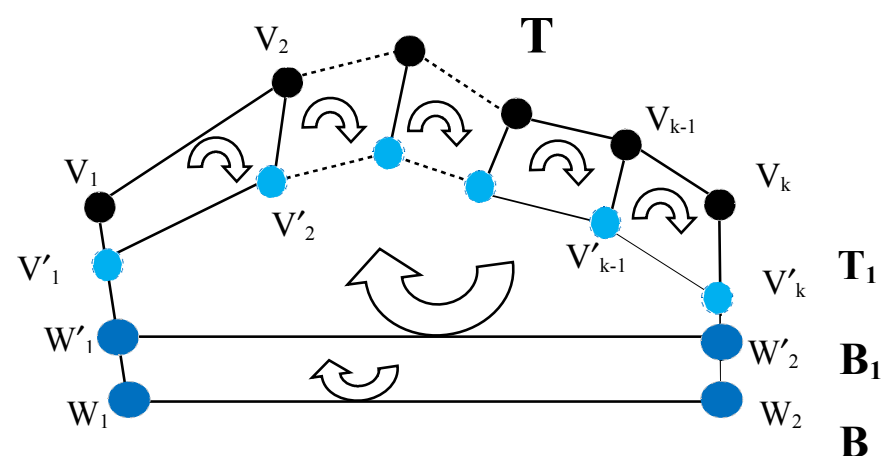

Fig. 7. Final faces after decomposition of a primitive face

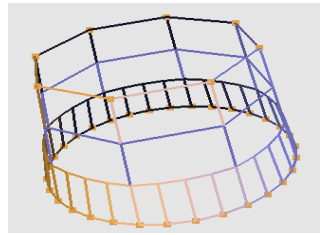

Fig. 8. Polyhedrons for incompatible contours

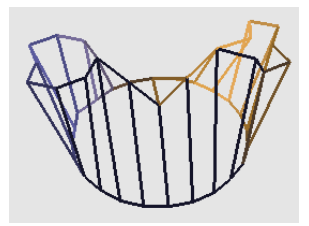

Fig. 9. Polyhedrons for branching contours

\section{Results and Discussions}

A large number of results have been tested in this work for contours having branching problems. The data structure implemented supports recursive Catmull-Clark subdivision. $\mathrm{C}++$ and OpenGL have been used in current formulation. Figure 9 shows the constructed polygons for a branching problem in which a layer has two contours and they correspond to a contour an adjacent layer. Fig. 10 shows a wire frame surface obtained by the present algorithms after smoothening by reversible Catmull-Clark subdivision. In this test result, there are three contours at a layer and they are corresponding to a contour at an adjacent layer. 


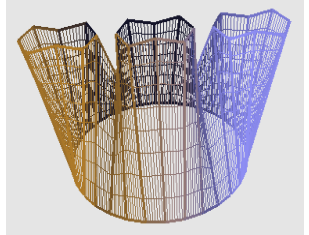

Fig. 10. Wire frame surface with constructed polyhedrons

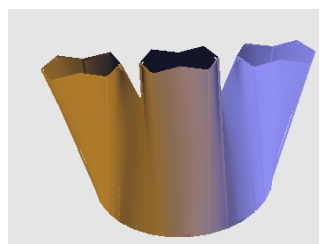

Fig. 11. Shaded branching surface

Fig 11 shows the shaded surface for the same test result. Another test result is shown in Fig 12 which has three contours at a layer and two contours an adjacent layer. Fig 13 shows the shaded surface for the test result shown in Fig 12. Fig 14 shows another test result-having contours at seven layers, the first and the last layer having two contours and it is correspondence with contours at adjacent layers. Fig. 15 shows the shaded surface for the test result shown in Fig 14. The technique given in $[5,6]$ can not directly handle the branching surface. The results given in [19] for branching are shifted towards the junction of the layer having single contour.

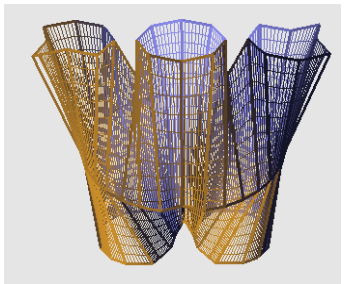

Fig. 12. Wire frame branching surfaces

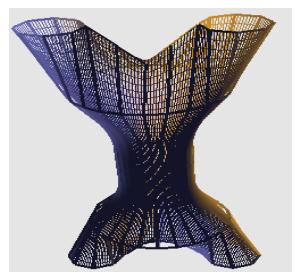

Fig.14. Subdivided wire frame branching surface

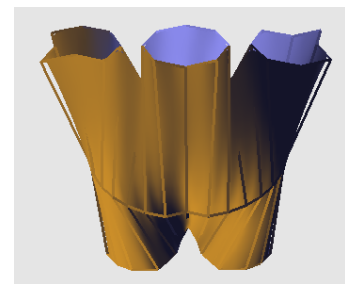

Fig. 13. Shaded surface \& constructed polyhedrons

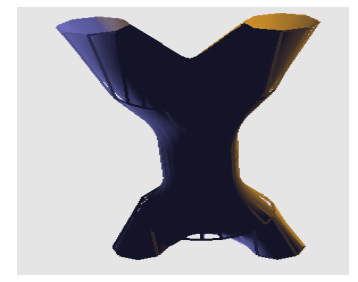

Fig. 15. Shaded branching surface with constructed polyhedrons

\section{Conclusions}

An algorithm has been developed to get a smooth surface from contours having branching problem. Three-dimensional composite curves have been obtained for the contours having branching problem on the basis of merging of the centre of the contours towards the center of adjacent contours. The polyhedrons have been constructed 
between the 3D composite curves and other contours. Two adjacent contours which are free from branching problem are selected for construction of polyhedrons. Reversible Catmull-Clark has been designed in the present formulation for the required smooth surface for better rendering. Different results for branching problem have been implemented.

\section{References}

1. Bajaj, C.L., Coyle, E.J., Lin, K.N.: Arbitrary topology shape reconstruction from planner cross sections. Graphical Models \& Image Proc. 58, 524-543 (1996)

2. Christiansen, H.N., Sederberg, T.W.: Conversion of complex contour line definitions into polygonal element mosaics. In: Computer Graphics (SIGGRAPH 1978 Proceedings), vol. 12, pp. 187-192 (1978)

3. Park, H., Kim, K., Lee, S.-C.: A Method for Approximate NURBS Curve Compatibility Based on Multiple Curve refitting. Computer Aided Design 32(20), 237-252 (2000)

4. Jha, K.: Energy based multiple refitting for skinning. International Journal of CAD/CAM 5(1), 11-18 (2005)

5. Jha, K.: Catmull-Clark Subdivision and Skinning of Incompatible Curves. In: International Conference on Trends in Product Life Cycle Modelling, Simulation and Synthesis (PLMSS 2006), pp. 83-90. IISc., Bangalore (2006)

6. Nasri, A., Abbas, A., Hasbini, I.: Skinning Catmull-Clark Subdivision Surfaces with Incompatible Curves. In: Proceeding of the 11th Pacific conference on Computer Graphics and applications, PG 2003 (2003)

7. Sederberg, T., Zheng, J., Bakenov, A., Nasri, A.: T-spline and T-NURCUS. ACM Transactions on Graphics, SIGGRAPH 22(3), 477-484 (2003)

8. Doo, D., Sabin, M.: Behaviour of recursive division surfaces near extraordinary points. Computer Aided design 10, 356-360 (1978)

9. Meyers, D., Skinner, S., Sloan, K.: Surfaces from contours. ACM Trans. On Graphics 11, 228-259 (1992)

10. Shinagawa, Y., Kunii, T.L.: The homotopy model: A generalized model for smooth surface generation from cross sectional data. Visual Computer 7, 72-86 (1991)

11. Ekole, A.B., Pyrin, F.C., Odet, C.L.: A triangulation algorithm from arbitrary shaped multiple planner contours. ACM trans. on Graphics 10, 182-199 (1991)

12. Jeong, J., Kim, K., Park, H., Cho, M., Jung, M.: B-Spline surface approximation to cross section using distance maps. Adv. Manuf. Techn. 15, 876-885 (1999)

13. Barequet, G., Shapiro, D., Tal, A.: Multilevel sensitive reconstruction of polyhedral surfaces from parallel slices. Visual Computer 16, 116-133 (2000)

14. Barequet, G., Sharir, M.: Piecewise-linear interpolation between polygonal slices. Comp. Vision \& Image Underst. 63, 251-272 (1996)

15. Goodman, T.N.T., Ong, B.H., Unsworth, K.: Reconstruction of $\mathrm{C} 1$ closed surfaces with branching. In: Farin, G., Hagen, H., Noltemeier, H. (eds.) Geometric Modelling, pp. 101115. Springer, London (1993)

16. Bedi, S.: Surface design using functional blending. CAD 24, 505-511 (1992)

17. Wang, H., Qin, K., Tang, K.: Efficient Wavelet Construction with Catmull-Clark Subdivision. Visual Computation 22, 874-884 (2006)

18. Loop, C., Schafer, S.: Approximating Catmull-Clark Subdivision Surfaces by Bicubic Patch, Technical Report, MST-TR-2007-44 (2007) 
19. Gabrielides, N.C., Ginnis, A.I., Kaklis, P.D., Karavelas, M.I.: G1-smooth branching surface contruction from cross sections. CAD 39(8), 639-651 (2007)

20. Jha, K.: Construction of branching surface from 2-D contours. International Journal of CAD/CAD 8 (2008)

21. Catmull, E., Clark, J.: Recursive Generated B-Spline Surfaces on Arbitrary Topological Meshes. The Journal of CAD and Application 1(1-4) (1978) 